\title{
A Doença Oncológica não deve ser um Fator Limitante para Admissão na UTI de Pacientes Submetidos a Cirurgias de Alto Risco*
}

\author{
Cancer Disease Should not be a Limitative Factor for \\ Admission of High Risk Surgical Patients to an Intensive Care Unit
}

Cristina P. Amendola', Samantha L. S. Almeida', Vanessa M. Horta ${ }^{1}$, Érica Sousa ${ }^{2}$,

Célio A. B. Gusmão², José M A. Sousa ${ }^{3}$, João M. Silva Júnior ${ }^{3}$, Ederlon Rezende ${ }^{4}$

\section{RESUMO}

JUSTIFICATIVA E OBJETIVOS: A doença oncológica per se é uma condição que muitas vezes influencia no tratamento dispensado ao paciente. O objetivo do presente estudo foi comparar o desfecho hospitalar de pacientes oncológicos e não oncológicos submetidos à procedimentos cirúrgicos eletivos com alto risco de óbito.

MÉTODO: Estudo de coorte prospectivo observacional, realizado em UTI de hospital terciário no período de 01 de abril a 31 de julho de 2005. Foram coletados dados demográficos, escore APACHE II, MODS,

1. Residente em Medicina Intensiva do Serviço de Terapia Intensiva Hospital do Servidor Público Estadual;

2. Médico Supervisor do Serviço de Terapia Intensiva Hospital do Servidor Público Estadual;

3. Médico Assistente do Serviço de Terapia Intensiva Hospital do Servidor Público Estadual;

4. Diretor do Serviço de Terapia Intensiva do Hospital do Servidor Público Estadual.

*Recebido do Serviço de Terapia Intensiva do Hospital do Servidor Público Estadual Francisco Morato de Oliveira - HSPE - FMO, São Paulo, SP

- Trabalho apresentado pela Dra. Cristina P Amendola para obtenção do Título de Especialista em Medicina Intensiva pela Associação de Medicina Intensiva Brasileira - AMIB.

Apresentado em 04 de maio de 2006

Aceito para publicação em 26 de junho de 2006

Endereço para correspondência:

Rua Pedro de Toledo, $18006^{\circ}$ andar - Vila Clementino

04039-901 São Paulo, SP

Fone/Fax (11) 5088-8146

E-mail: ederlon@sti-hspe.com.br

(C)Associação de Medicina Intensiva Brasileira, 2006 variáveis hemodinâmicas, laboratoriais e avaliadas complicações definidas como re-operação, necessidade de ventilação mecânica, transfusão sangüínea e uso de cateter de artéria pulmonar no pós-operatório desses pacientes. Todos foram acompanhados até alta ou o óbito hospitalar. Para variáveis numéricas foi utilizado o teste $t$ de Student e Mann-Whitney, para variáveis categóricas o teste do Qui-quadrado sendo considerado significativo o valor de $\mathrm{p}<0,05$.

RESULTADOS: Foram incluídos no estudo 119 pacientes, 43 não oncológicos e 76 oncológicos, 52,9\% eram do sexo feminino. A média de idade foi $65,1 \pm$ 14,1 anos, o escore médio de APACHE II 16,5 \pm 5,8 e a mediana do MODS de 3 (2-6). A duração mediana da intervenção cirúrgica foi de $5(3,3-7)$ horas e a mortalidade na UTI e hospitalar foram $10,9 \%$ e $25,2 \%$, respectivamente. Os pacientes oncológicos apresentaram maiores tempos de internação hospitalar e de internação antes da cirurgia, sendo estes resultados estatisticamente significativos. A mortalidade hospitalar dos pacientes oncológicos não foi superior a dos pacientes sem neoplasia $(22,4 \%$ versus $30,2 \%, p=0,32$ ).

CONCLUSÕES: Nesta observação os pacientes oncológicos, submetidos à procedimentos cirúrgicos de alto risco, apresentaram mortalidade semelhante aos pacientes não oncológicos com gravidade de doença similar.

Unitermos: cirurgia de alto risco, mortalidade, paciente oncológico, prognóstico

\section{SUMMARY}

BACKGROUND AND OBJECTIVES: Oncologic diseases are conditions that have influence in the tre- 
atment offered to affected patients. The aim of this study was to compare hospitalar outcome of oncologic and non oncologic patients submitted to high risk elective surgery.

METHODS: Prospective, observational cohort study realized in an ICU of a tertiary hospital during the period between 04/01/2005 and 07/31/2005. Demographic data, APACHE II and MODS scores and laboratorial and hemodynamic variables were collected and complications like re-intervention need for mechanical ventilation, red blood cell transfusions and pulmonary artery catheter use during the post-operative period were evaluated. All patients were followed until hospital discharge or death. T student and Mann Whitney tests were used to compare numerical variables. Chi-square test was used to compare categorical variables. A $p<0.05$ was considered as significant.

RESULTS: 119 patients were included in the study. 43 were oncologic and 76 were non-oncologic. $52.9 \%$ were female. Mean age was $65.1 \pm 14.1$ years. Mean APACHE II score was $16.5 \pm 5.8$ and MODS median was 3 (2-6). Median length of surgery was 5 (3.3-7) hours and ICU and hospital mortality were $10.9 \%$ and $25.2 \%$, respectively. Oncologic patients had greater length of hospital stay and length of stay before surgery. These results were statistically significant. Hospital mortality of oncologic patients was not greater than non-oncologic patients $(22.4 \%$ versus $30.2 \%, p$ $=0.32$ ).

CONCLUSIONS: In this series, oncologic patients submitted to high risk surgery had the same mortality rate as non-onconlogic patients with similar disease severity.

Key Words: high risk surgery, mortality, oncologic patient, outcome

\section{INTRODUÇÃO}

As últimas décadas se caracterizaram importantes por avanços no diagnóstico precoce e no manuseio de pacientes com câncer, o que resultou em redução de $20 \%$ na mortalidade geral. Um dos fatores que contribuíram para essa redução foi a terapêutica avançada em unidades de terapia intensiva (UTI) ${ }^{1-3}$. Apesar dessa redução a taxa de mortalidade ainda permanece alta, cerca de $45,7 \%{ }^{4}$.

Nos EUA, em 2001, a mortalidade por neoplasia era de 500.000 pessoas, mais de 1500 por dia. O câncer é a segunda causa de morte, perdendo apenas para as doenças cardiovasculares. Uma em cada quatro mortes em 2001 teve como causa doença neoplásica. Além disso, mais de um milhão de novos casos foram diagnosticados em 2000, representando um custo de tratamento de 180 bilhões de dólares/ ano $0^{5,6}$.

Mais de 40 milhões de procedimentos cirúrgicos são realizados nos EUA e na Inglaterra a cada ano, com grande parte deles envolvendo pacientes de moderado ou alto risco cirúrgico. A mortalidade observada em pacientes de alto risco varia de $9,7 \%$ nos EUA a $35,9 \%$ na Inglaterra ${ }^{7,8}$. Nos EUA, em 2001, a estimativa dos tipos de câncer para os quais as cirurgias aparecem como uma das formas de tratamento foi expressiva: cânceres mama 182.800 , pulmão 164.100 , colo-retal 125.000 , renal 30.800 , cabeça e pescoço 30.000, pâncreas 29.200, hepático 25.000, ovário 23.100, gástrico 22.000, tireóide 19.500, próstata 18.900, intra-encefálico 17.200 e esofágico $13.000^{9}$.

O diagnóstico de neoplasia parece ser um agravante para admissão do paciente na UTI. Em estudo francês que avaliou a admissão de pacientes oncológicos na UTI, verificou-se que $49 \%$ são recusados por apresentarem a doença ${ }^{4}$. Em virtude dos avanços na sobrevida de pacientes oncológicos, cabe ao intensivista conhecer melhor esse grupo de pacientes e definir, de maneira objetiva e mais adequada possível, o grau de investimento a ser realizado. Além disso, discernir os que se beneficiarão de uma intervenção agressiva daqueles para os quais são indicados apenas cuidados paliativos também é fator importante.

Dessa forma, o presente estudo teve como objetivo avaliar a sobrevida hospitalar de pacientes oncológicos em relação aos não oncológicos, submetidos a procedimentos cirúrgicos eletivos de alto risco.

\section{MÉTODO}

Após aprovação pelo Comitê de Ética e Pesquisa, que dispensou o termo de consentimento livre e esclarecido, foi realizado um estudo de coorte observacional durante período de 01 de abril 31 de julho de 2005, em unidade de terapia intensiva de hospital público terciário.

Foram coletados consecutivamente dados de 119 pacientes submetidos a intervenções cirúrgicas eletivas, com intenção de cura e idade maior que 18 anos encaminhados para pós-operatório na UTI. Os pacientes 
submetidos a procedimentos cirúrgicos cardiovasculares foram excluídos.

Os piores valores das variáveis nas primeiras 24 horas da admissão na UTI foram coletados para a realização de escores APACHE II ${ }^{10}$ (Acute Physiology and Chronic Healt Evalution II) e MODS ${ }^{11}$ (Multiple Organ Dysfunction Score). Outros dados como análise demográfica, tempo do procedimento, tempo de internação na UTI e hospitalar, foram avaliados durante o pós-operatório imediato (POI), que compreendia desde a admissão na UTI até às 6 horas do dia seguinte. Os pacientes foram acompanhados até alta ou óbito hospitalar e o pesquisador não exercia qualquer influência nas decisões dos médicos que assistiram o paciente.

Para análise estatística, os dados foram demonstrados como média \pm desvio-padrão, mediana (intervalo interquartil) e porcentagens. As variáveis com distribuição normal foi utilizado o teste $t$ de Student, variáveis sem distribuição normal o teste de MannWithney e variáveis categóricas o teste do Qui-quadrado; $p<0,05$ (bicaudal) foi considerado significativo. As estimativas de curvas de sobrevida no hospital foram calculadas utilizando regressão pelo método de Cox.

\section{RESULTADOS}

A média da idade foi de 65,1 anos, não havendo diferença entre os sexos. A duração mediana dos procedimentos cirúrgicos foi de 5 horas e o tempo de internação hospitalar 20 dias. Durante a internação hospitalar 25,2\% dos pacientes evoluíram para óbito. As características dessa população se encontram na tabela 1.

Tabela 1 - Características dos Pacientes

\begin{tabular}{lc}
\hline Variáveis & \\
\hline Idade (anos) & $65,1 \pm 14,1^{*}$ \\
Sexo feminino (\%) & 52,9 \\
APACHE II (pontos) & $16,5 \pm 5,8$ * \\
MODS (pontos) & $3(2-6)$ \\
Duração da cirurgia (horas) & $5(3,3-7)$ \\
Tempo de internação na UTI (dias) & $1(1-3,0)$ \\
Tempo de internação hospitalar (dias) & $20(10-35)$ \\
Metástase à distância (\%) & 15 \\
Mortalidade na UTI (\%) & 10,9 \\
Mortalidade hospitalar (\%) & 25,2 \\
\hline
\end{tabular}

* Valores expressos em Média \pm DP
A maioria dos pacientes $(51,2 \%)$ foi submetida a intervenções cirúrgicas do aparelho digestivo. Todas as cirurgias apresentavam intenção de cura (Tabela 2).

Tabela 2 - Cirurgias Realizadas por Especialidades nos Dois Grupos de Pacientes

\begin{tabular}{lcccc}
\hline Cirurgias & $\begin{array}{c}\text { Não } \\
\text { Oncológicos } \% \\
(\mathrm{n}=43)\end{array}$ & $\begin{array}{c}\text { Oncológicos } \% \\
(\mathrm{n}=76)\end{array}$ & $\begin{array}{c}\text { Total \% } \\
(\mathrm{n}=119)\end{array}$ & $\mathrm{p}$ \\
\hline Aparelho & $51,2(22)$ & $48,7(37)$ & $49,5(59)$ & 0,945 \\
digestivo & $9,3(4)$ & $13,2(10)$ & $11,8(14)$ & 0,741 \\
Torácica & $25,6(11)$ & $10,5(8)$ & $15,9(19)$ & 0,058 \\
Ortopédica & $2,3(1)$ & $11,8(9)$ & $8,4(10)$ & 0,136 \\
Ginecológica & $7,0(3)$ & $7,9(6)$ & $7,6(9)$ & 0,769 \\
Urológica & $4,7(2)$ & $7,9(6)$ & $6,7(8)$ & 0,766 \\
Outras* & & & &
\end{tabular}

*Cabeça e pescoço, neurologia, otorrinolaringologia, transplante.

Comparando os grupos de pacientes oncológicos e não oncológicos, o tempo de hospitalização antes da cirurgia e a duração de internação hospitalar foram maiores no grupo de pacientes oncológicos. Entretanto, a diferença de mortalidade na UTI e hospital não foi significativa. (Tabela 3), o que foi corroborado pela análise da curva de sobrevida pelo método de regressão de Cox, entre pacientes não oncológicos e oncológicos. (Figura 1)

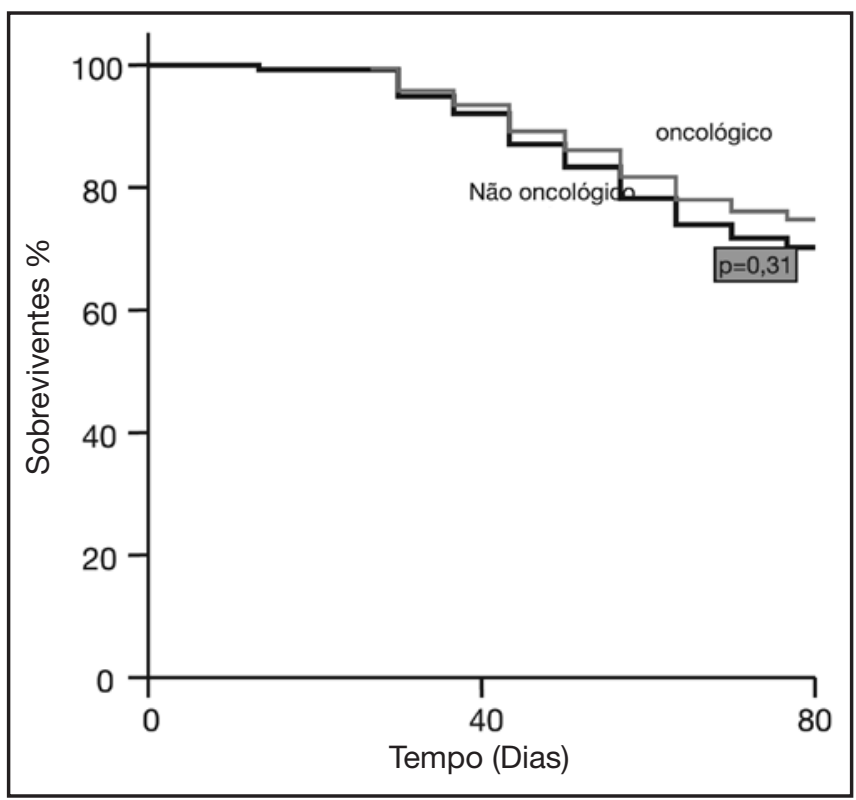

Figura 1 - Curva de Sobrevida pelo Método de Regressão Cox entre Pacientes Não Oncológicos e Oncológicos 
AMENDOLA, ALMEIDA, HORTA E COL.

Tabela 3 - Variáveis Hemodinâmicas, Laboratoriais e Escores entre os Dois Grupos Estudados

\begin{tabular}{lccc}
\hline Variáveis & $\begin{array}{c}\text { Oncológicos } \\
(\mathrm{n}=76)\end{array}$ & $\begin{array}{c}\text { Não Oncológicos } \\
(\mathrm{n}=43)\end{array}$ & $\mathrm{p}$ \\
\hline Idade (anos) & $66,6 \pm 11,2$ & $62,5 \pm 17,9$ & 0.13 \\
Sexo feminino (\%) & 47,4 & 62,8 & 0,10 \\
APACHE II (pontos) & $16,1 \pm 4.6$ & $17,1 \pm 7,5$ & 0.34 \\
MODS (pontos) & $3(0-12)$ & $3(0-11)$ & 0,80 \\
Duração da cirurgia (horas) & $5,3(1,3-10,2)$ & $4,5(1,0-17,0)$ & 0,41 \\
Tempo de internação UTI (dias) & $1(0.5-32)$ & $1(0,5-61)$ & 0.21 \\
Tempo de internação hospitalar (dias) & $20(2-166)$ & $15(4-85)$ & 0,03 \\
Tempo de internação antes da cirurgia (dias) & $8,0(0,5-139)$ & $6,0(0,5-61)$ & 0,03 \\
Pressão arterial média (mmHg) & $72,8 \pm 12,3$ & $70,0 \pm 15,7$ & 0.28 \\
Hematócrito (\%) & $33 \pm 5,8$ & $32,2 \pm 6,7$ & 0,53 \\
Lactato arterial (mmol/L) & $2,3(0,5-8,6)$ & $2,1(0,8-6,8)$ & 0.79 \\
Freqüência cardíaca (bpm) & $108,5 \pm 19.8$ & $110,6 \pm 21,1$ & 0.64 \\
Glicemia (mg/dL) & $155,6 \pm 48$ & $159,6 \pm 53,9$ & 0,67 \\
Balanço hídrico no pós-operatório imediato & $1873,-1702 / 6527)$ & $2500(-2086 / 7732)$ & 0,16 \\
Mortalidade hospitalar (\%) & 22,4 & 30,2 & 0,34 \\
Mortalidade UTI (\%) & 7,9 & 16,3 & 0,16 \\
Re-operação (\%) & 10,5 & 14,6 & 0,51 \\
Intubação traqueal no pós-operatório imediato (\%) & 2,6 & 2,3 & 0,91 \\
VMNI no pós-operatório imediato (\%) & 3,9 & 9,3 & 0,23 \\
Cateter de artéria pulmonar (\%) & 0 & 1,4 & 0,29 \\
Transfusão de hemácias no pós-operatório imediato (\%) & 3,9 & 0 & 0,18 \\
Tempo de ventilação mecânica no pós-operatório imediato (dias) & $0,5(0,5-288)$ & $0,5(0,5-450)$ & 0,55 \\
\hline
\end{tabular}

VMNI - ventilação mecânica não-invasiva.

\section{DISCUSSÃo}

A taxa de sobrevida em cinco anos em pacientes com neoplasia é de $60 \%{ }^{5}$. Em contrapartida, a mortalidade vem diminuindo consideravelmente com os novos avanços $^{1}$. O progresso do tratamento é atribuído ao suporte de terapia intensiva e as cirurgias, que são menos invasivas e priorizam melhor qualidade de vida ${ }^{5}$. Essas novas informações exigem melhor abordagem por parte dos médicos intensivistas na hora de disponibilizar leitos para o tratamento intensivo. Sendo assim, a decisão quanto à triagem de pacientes é sempre um grande desafio, pois freqüentemente há poucas informações disponíveis sobre o caso. Além disso, a tentativa de predizer a evolução de pacientes individualmente através de escores que avaliam gravidade (APACHE, SAPS ou MPM) apresenta algumas limitações ${ }^{8,9,12}$, mesmo escores específicos de câncer ${ }^{13}$.

No presente estudo foram avaliados dois grupos, oncológicos e não oncológicos, de pacientes de cirurgias eletivas, considerados de alto risco, que apresentaram mediana de 5 horas no tempo de cirurgia e necessitaram pós-operatório na UTI. Além disso, a porcentagem de pacientes com metástase à distância do grupo oncológico representou $15 \%$, porém não foi possível determinar as metástases peri-lesão neoplásica, o que provavelmente pelo porte cirúrgico pode-se estimar que praticamente a maioria dos pacientes apresentava.

Poucos estudos avaliaram o prognóstico deste grupo de pacientes. A literatura apresenta dados divergentes quanto à mortalidade de pacientes oncológicos, desde $75 \%$ em menos de três meses após alta da UTI ${ }^{14,15}$ até estimativas de $63 \%, 57 \%, 53 \%$ e $51 \%$ de taxa de sobrevida para todos os tipos de cânceres, respectivamente em 5, 10, 15 e 20 anos $^{14}$. No entanto, para esse grupo de pacientes ainda há restrições para suporte intensivo.

Neste estudo os procedimentos cirúrgicos do aparelho digestivo foram predominantes nos dois grupos, concordantes com a prevalência de câncer nessa localização ${ }^{9}$ e de acordo com dados do Instituto Nacional do Câncer (INCA). Dessa forma, as cirurgias realizadas apresentaram portes cirúrgicos muito semelhantes, o que permite fazer comparações entre os grupos. A exceção se faz em relação às cirurgias vasculares que só estiveram presentes em pacientes não oncológicos, por este motivo foram excluídas do estudo. 
Os grupos de pacientes oncológicos e não oncológicos apresentaram a mesma idade, sexo, escores de gravidade APACHE II e MODS, além de mesmo tempo de cirurgia, sugerindo que somente o câncer poderia ser o fator agravante da evolução pós-cirúrgica. Ainda assim, no pós-operatório imediato as variáveis laboratoriais, hemodinâmicas e intercorrências, como necessidade de re-operação, ventilação mecânica e transfusão sangüínea tiveram a mesma incidência.

Entretanto, o tempo de internação antes da cirurgia, assim como a permanência hospitalar foi maior nos pacientes oncológicos. Isto corrobora dados da literatura em que este grupo de pacientes, quando operados, necessitam de preparo adequado além de, no pósoperatório, permanecerem por maior tempo internados no hospital com o intuito de tratamento complementar como quimioterapia, radioterapia ou observação mais rigorosa na evolução do câncer ${ }^{5}$.

Nesta analise o ponto fundamental é que a mortalidade hospitalar nos dois grupos não foi diferente, inclusive com tendência a menor mortalidade em pacientes oncológicos.

De fato pacientes oncológicos não apresentaram piores prognósticos, mesmo sendo considerados graves. Dessa forma, a avaliação inicial destes pacientes deve ser mais cuidadosa. Thiery e col. observaram que a opinião entre os especialistas da área de oncologia e terapia intensiva é discordante em relação ao prognóstico, o intensivista tende a subestimar uma melhor evolução ${ }^{4}$. No entanto em outras doenças como a sepse grave ou choque séptico, onde a taxa de mortalidade é elevada, até $70 \%$ em um ano ${ }^{16,17}$, não há tal restrição pelos intensivistas em relação ao suporte. Soares e col. demonstraram que a idade avançada, a gravidade das disfunções e a presença de neoplasia não controlada são variáveis importantes a se considerar no prognóstico de pacientes com câncer ${ }^{18}$. Outro fator interessante deste estudo foi que a avaliação até 80 dias de internação não mostrou diferença entre os grupos em relação à mortalidade, portanto a doença oncológica per se não foi fator independente de mau prognóstico.

Todavia, não se avaliou a qualidade de vida que estes pacientes apresentaram após as cirurgias e a mortalidade em longo prazo, já que avaliações com previsão de cura da maioria das doenças oncológicas são após 5 anos $^{5}$. Porém determinar esses aspectos não faz parte somente da avaliação médica, mas de uma decisão conjunta com o paciente e seus familiares levando em conta os seus desejos e expectativas.

Dessa forma os médicos intensivistas devem estar familiarizados com os novos avanços terapêuticos também nesta área oncológica no intuito de não limitar o suporte necessário nesta condição.

O paciente oncológico apesar de maior morbidade caracterizada por permanência hospitalar prolongada apresenta desfecho final similar aos pacientes não oncológicos.

Não se pode atribuir à doença oncológica isoladamente pior prognóstico, portanto, durante o processo de triagem para admissão na UTI, a doença oncológica com possibilidade de intervenção cirúrgica não deve ser considerada como limitante, especialmente para o suporte pós-operatório.

\section{REFERÊNCIAS}

01. Azoulay E, Afessa B - The intensive care support of patients with malig nancy: do everything that can be done. Intensive Care Med. 2006;32:3-5.

02. Azoulay E, Recher C, Alberti $\mathrm{C}$ et al - Changing use of intensive care for hematological patients: The example of multiple mieloma. Intensive Care Med, 1999;25:1395-1401.

03. Larche J, Azoulay E, Fieux F et al - Improved survival of critically ill cancer patients with septic shock. Intensive Care Med, 2003;29:1688-1695.

04. Thiery E, Azoulay E, Darmon M et al - Outcome of cancer patients considered for intensive care unit admission: A hospital wide prospective study. J Clin Oncol, 2005;23:4406-4413.

05. Schmiesing CA, Fischer SP - The preoperative assessment of the cancer patient. Cur Opin Anaesthesiol, 2001;14:721-729.

06. Bennett GE, Hyam JA, Shaefi S et al - Comparison of P-POSSUM riskadjusted mortality rates after surgery between patients in the USA and the UK. Br J Surg, 2003;90:1593-1598.

07. Mangano DTE - Peroperative cardiac morbidity. Anesthesiology, 1990;72:153-184.

08. Graf J, Janssens $U$ - Still a black box: What do we really know about the intensive care unit admission process and its consequences? Crit Care Med, 2005;33:901-903.

09. Dragsted L, Jörgensen J, Jensen NH et al - Interhospital comparisons of patient outcome from intensive care: importance of lead-time bias. Crit Care Med, 1989;17:418-422.

10. Knaus WA, Zimmerman JE, Wagner DP et al - APACHE, acute physiology and chronic health evaluation: a physiologically based classification system. Crit Care Med, 1981;9:591-597.

11. Marshall J, Cook D, Chirstou N et al - Multiple Organ Dysfunction score: a reliable descriptor of a complex clinical outcome. Crit Care Med, 1995;23:1638-1652.

12. Goldhill DR, Withington PS - The effect of casemix adjustment on mortality as predicted by APACHE II. Intensive Care Med, 1996; 22:415-419.

13. Soares $\mathrm{M}$, Fontes $\mathrm{F}$, Dantas $\mathrm{J}$ et al - Performance of six severity-of-illness scores in cancer patients requiring admission to the intensive care unit: a prospective observational study. Critical Care, 2004;8:R194-R203.

14. Nelson JE, Meier DE, Oei EJ et al - Self-reported symptom experience of critically ill cancer patients receiving intensive care. Crit Care Med, 2001;29:277-282.

15. Schapira DV, Studnick J, Bradham DD et al - Intensive care survival, and expense of treating critically ill cancer patients. JAMA, 1993; 269:783-786.

16. Quartin AA, Schein RM, Kett DH et al - Magnitude and duration of the effect of sepsis on survival. Department of Veterans Affairs Systemic Sepsis Cooperative Studies Group. JAMA, 1997;277:1058-1063.

17. Veterans Administration Systemic sepsis Cooperative Study Group: Effect of high dose glucocorticoid therapy on mortality in patients with clinical signs of systemic sepsis. N Engl J Med, 1987;317: 659-665.

18. Soares M, Salluh JI, Rocco JR et al - Fatores prognósticos em pacientes com neoplasias hematológicas gravemente enfermos. RBTI, 2005;17:170-175. 